\title{
Modeling Liquid Terminal Operation and Service System in a Port Using Linier Matrix Algebraic Approach
}

\author{
Anwarudin ${ }^{1}$, Ofyar Z. Tamin ${ }^{2}$, Gatot Yudoko ${ }^{3}$, Muhammad Sutarno ${ }^{4}$ \\ ${ }^{I}$ (School of Architecture, Planning and Policy Development, Bandung Institute of Technology, Bandung, \\ Indonesia) \\ ${ }^{2}$ (Department of Civil Engineering, Bandung Institute of Technology, Bandung, Indonesia) \\ ${ }^{3}$ (School of Business Management, Bandung Institute of Technology, Bandung, Indonesia) \\ ${ }^{4}$ (Faculty of Industrial Technology, Bandung Institute of Technology, Bandung, Indonesia)
}

\begin{abstract}
Performance parameters of a terminal service operation in port can be determined using numerous methods available in the literature. The terminal service operations mostly anaytically modelled based on queueing theory formulation, fuzzy methods or game theory approach. In some cases, simulation models frequently used to get deeper and more details performance measures of the system. In this paper, we use analytical matrix algebraic approach to represent terminal operation and customer's service system in port. In this approach, we try to make broader disaggregation of customer's type in the system, based on customer service time and customer's service cost. Our model is amenable, especially when waiting time was considered costly for a part of customer, whereas others concern mostly on service cost. Our model has been proved satisfied to analyze service operation for liquid terminal handling CPO cargo in Port of Dumai, Indonesia. Further applications of the model are open for similar service system.
\end{abstract}

Keywords - Port operation, liquid terminal, matrix algebraic approach

\section{INTRODUCTION}

Most of old ports originally built with single multipurpose terminal serving any kind of ship and handling any kind of cargo, including passenger embarkment/disembarkment. Any commodities and passengers have to use the same berth and access way for loading or unloading operation of the ship. The multipurpose terminal in port usually equipped with limited and general purpose cargo handling facilities, such as fork-lift and mobile crane. In case any cargo needs special cargo operation equipments, then the shippers shall provide it themselves on their own responsibility.

One kind of cargo that required special equipment is liquid cargo. The liquid cargo needs a big storage/loading tank near the wharf, pipelines, pumps and pig launcher to clean the pipelines after cargo loading/unloading. If the cargo was handled in multipurpose terminal, then the shipper should provide and operate those facilities on their own responsibility. Every shipper that needs similar facilities has to provide it themselves or use others.

If this liquid cargo growth significantly, and the multipurpose terminal was still used to handle the cargo, there are several problems are raising in the terminal. The technical and operational problems could be identified as follows:

a. Since many facilities were built and operated for the shipper interest only, there will be too many pipelines on access way and it could be disturb a smooth services operation for other kind of cargo, and vice versa;

b. The dead load coming from numerous pipeline's weight and dynamic load of pump pressures could be dangerous for trestle or wharf structure since they were not originally designed for such loads;

c. Pipe dimension, pumps power, and tank storage position which located far and more far away from berthing point of the ship caused loading/unloading speed vary significantly and tends to operated slowly;

d. Cargo arrival to the port was not synchronously scheduled according to the arrival plan of the ship causing the ship has to wait longer in the wharf.

Those problems will decrease port level services, caused severe congestion and additional high cost, both for liquid and general cargo as well. Ships will be queued longer in front of the wharf and customers will be suffered extra cost in transporting their goods. To avoide this severe situation, port operator shall provide a dedicated liquid terminal to serve the liquid cargo shipper.

Due to high investments needed, the liquid terminal facilities have been designed with limited capacity causing each customers have to be wait if the server are busy. Therefore, cost of waiting can not be avoided for almost all customers. Unfortunately, the customers of the liquid terminal are tanker ships which actually have different cost of waiting according to their size. Some big customers will be very burdened with heavy waiting cost per time unit, whereas some small customers were not considered it at all. 
The customers of the liquid terminal could be divided into three categories based on their costs in the port. First, there are small ships that loaded or unloaded a low volume of liquid cargo. Due to their small size, the waiting costs of ships are low and they have served fastest in the system. Therefore, their cost are smallest comparing to other classes. Second, on the contrary, there are big ships with high volume of liquid cargo. Of course they have very high waiting cost due to their big size and a lot of volume cargo that they should be carried. Unfortunately, this kind of shipper have to be served longest since they brought much more cargo. Hence, their cost in port are very high because they have to pay more both on cargo operations and ship waiting cost. The last category, are the ships in between.

In order to serve all class of the customers, the service system require a server that can serve with different mean service time for each class of customer. In this paper, we develop the model also to represents a server that could served two customers simultanously. Hence, we have a server with two service stations. The most important characteristic of the server is that each of the two service station has several phases with different service time and different service cost to served each class of customers.

\section{LITERATUR REVIEW}

In queueing theory, a model of server with quantification of customer behavior was started by Naor [1] which show induction of tolls as a mechanism of customer arrival control to the queuing system. Naor said that each customer will only consider his waiting time when decide to enter the system. He proved that they will not taking account to the impact of their decision to the next customers which will experienced longer waiting time.

Stidham [2] said that Naor's model is a shifting paradigm since it introduces a consideration of feedback on queuing system. An arrival process of customers that normally treats as exogenous process could be affected by congestion within the system itself. Naor's model also gives a brightening idea on customer's adaptive behavior to optimize their utility within the system which in turn leads to the idea of controlling the system through pricing mechanism. Knudsen [3] broadens Naor's work for multi-server system and nonlinear waiting cost function. Bradford [4] discussed a controlling mechanism that involves pricing and policy to assign customers to the server in a congested system. If customers owned private information regarding their waiting cost, system administrator that wants to maximize the benefit of his facilities could be use incentive compatible pricing schemes. Bradford model introduced incentive compatible pricing and route scheme for server capacity allocation.

Brouns [5] studied similar but simpler models with routing and admission control approach and single customer class. Recent work came from Ahn and Lewis [6] that considers joint routing and allocation policies in a two station parallel of queuing network. Their model includes routing costs and expansion for super-additive or sub-additive collaboration service rates. Within super-additive case, they found that optimal policy is exactly follow general intuition which should be done with clearing the higher cost first and never routing the customer to the high cost. In other case (sub-additive) they found that there is an inherent trade-off between serving faster and reducing costs at a high cost station. This trade-off and the fact that the routing can balance the workload will complicate the structure of optimal policy of the system.

Lipsky [7] has modeling two servers worked in a closed system using linier algebraic queueing theory approach. The system consists of two servers with different characteristics. The main server has two service stations and each of them has similar number of phases representing different service time distributions, whereas the other is simply a single exponential server which worked as a feeder for the main server. Our model was a modification of Lipsky system with several important characteristic discrepancies. First, we are working on open system with infinite customers instead of closed system with limited unchanged population number of customers. Second, we assumed that each customer, upon completion of service in one phase, will leave the system permanently. Even if the same customer returns into the system later, he acts as a different customer entity. In this case, we can eliminate state transition matrices that required on Lipsky's. In that view, our model is simpler. Third, we have single server, while there are two series of servers in Lipsky's work. Although our system has many different characteristics, we use similar approach and notation on his paper for consistency.

\section{MODEL FORMULATIONS}

We derived our problem to a model of a queueing system. The system consists of a service system with two different groups of server and two different kinds of customer. The service time in this server are assumed to be exponentially distributed. By assuming liquid shipper come to the port can be described with Poisson distribution, they will be separated and created two different Poisson distribution.

In order to get analytic solutions, we defined that the server in the system have two service station. Hence, it can serve two customers simultaneously. We assumed that the number of customers for each class follow Poisson distributions, while service time on all server assumed to be exponentially distributed. Every group of customers has their own service time distributions. The mean service time distributions for each group 
of customers will be represented by phases in each service station. All service station will have the same phases. Hence, customers are serve in the same phases on the same server, regadless which service station he uses.

In order to get steady state solution of the system, first we have to define all notations, vectors and matrices that will be used in this model in following definitions:

1. An entrance vector, denoted by a row vector $\mathbf{p}$, is representing the probabilities that a customer, upon entering the system will go to any phase $i$, where $i$ represent one phase available in the system. The probabilities of a customer to enter each phase $i$ will be represented by each element of $\mathbf{p}$, denoted by $\mathbf{p}_{i}$.

2. The dimension of state space $\boldsymbol{\Xi}_{\mathbf{1}}$ in the system, representing the number of phases in the system when there are $n \leq 1$ customer, will be denoted by $\mathrm{D}(1):=m$. Whereas the number of elements of state space $\boldsymbol{\Xi}_{\mathbf{2}}$, represents the system state probabilities when there are $n \geq 2$ customers in the system, will be defined as $\mathrm{D}(2)$ $:=m(m+1) / 2$.

3. The column vectors $\varepsilon_{1}^{\prime}$ and $\varepsilon_{2}^{\prime}$ are vectors which all entries 1 . The dimension of the vectors are $\mathrm{D}(1)$ and $\mathrm{D}(2)$ respectively.

4. The completion matrix $\mathbf{M}_{\mathbf{2}}$ is a matrix where the $i$-th diagonal elements, denoted by $\left[\mathbf{M}_{2}\right]_{i i}$, represents the probability rate of leaving state $i$ by a service completion in the system, whereas all others entries in the matrix will be zero. All diagonal elements will be formulated as $\left[\mathbf{M}_{2}\right]_{i i}:=\mu_{\mathrm{i} 1}+\mu_{\mathrm{i} 2}$. The completion matrix for state space $\boldsymbol{\Xi}_{1}$, represents the service completion in the system when there are $n \leq 1$ customer, is denoted by $\mathbf{M}_{1}$ and formulated as $\left[\mathbf{M}_{1}\right]_{i}:=\mu_{\mathrm{i}}$. The dimension of $\mathbf{M}_{\mathbf{1}}$ and $\mathbf{M}_{\mathbf{2}}$ completion matrices will be $\mathrm{D}(1) \times \mathrm{D}(1)$ and $\mathrm{D}(2) \times \mathrm{D}(2)$ respectively.

5. $\mathbf{Q}_{2}$ defined as a state demotion matrix that has a dimension of $D(2) \times D(1)$. The matrix represents probabilities to put the system into internal state with one customer, upon customer leaving off the system, given that it has two customers previously. The state demotion matrix with 4 phases in each service station will be in following formulation:

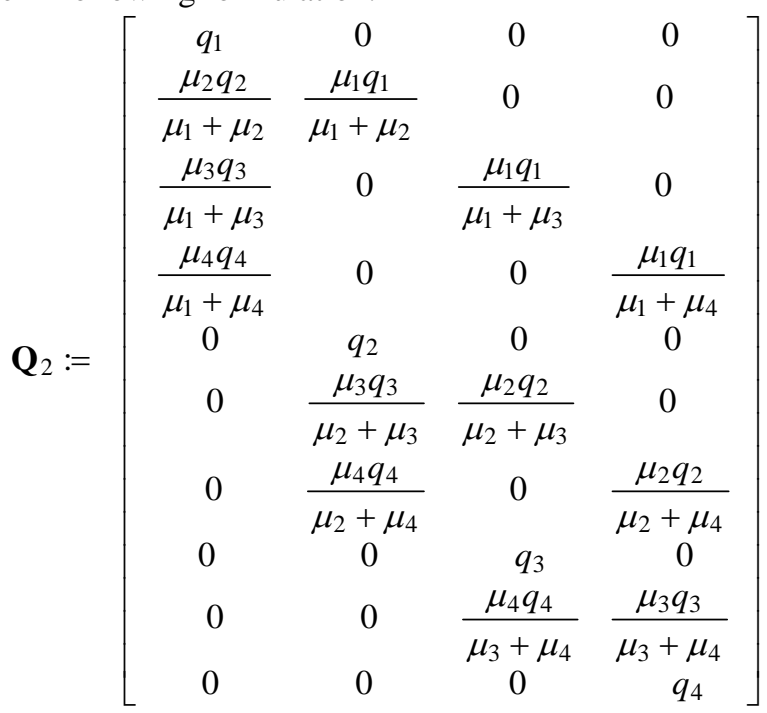

6. $\mathbf{R}_{2}$ defined as a state promotion matrix that has a dimension of $\mathrm{D}(1) \times \mathrm{D}(2)$. The matrix represents the probabilities to put the system in internal state with two customers, upon arrival of a customer into the system, given that it has one customer previously. The state promotion matrix for any system with 4 phases in each service station will be in following formulation:

$$
\mathbf{R}_{2}:=\left[\begin{array}{cccccccccc}
p_{1} & p_{2} & p_{3} & p_{4} & 0 & 0 & 0 & 0 & 0 & 0 \\
0 & p_{1} & 0 & 0 & p_{2} & p_{3} & p_{4} & 0 & 0 & 0 \\
0 & 0 & p_{1} & 0 & 0 & p_{2} & 0 & p_{3} & p_{4} & 0 \\
0 & 0 & 0 & p_{1} & 0 & 0 & p_{2} & 0 & p_{3} & p_{4}
\end{array}\right]
$$

7. The state transition matrices, denoted by $\mathbf{U}_{1}(\boldsymbol{N})$ and $\mathbf{U}_{2}(\boldsymbol{n})$, represent state transitions of the system for state spaces $\boldsymbol{\Xi}_{1}$ and state space $\boldsymbol{\Xi}_{1}$ respectively. The matrices could be listed as follows:

$$
\begin{aligned}
& \mathbf{U}_{1}(N):=\left[\mathbf{I}_{1}+\frac{1}{\lambda} \mathbf{M}_{1}-\mathbf{R}_{2} \mathbf{U}_{2}(N-2) \mathbf{M}_{2} \mathbf{Q}_{2}\right]^{-1} \\
& \mathbf{U}_{2}(1):=\left[\mathbf{I}_{2}+\frac{1}{\lambda} \mathbf{M}_{2}-\mathbf{Q}_{2} \mathbf{R}_{2}\right]^{-1}
\end{aligned}
$$


$\mathbf{U}_{2}(n):=\left[\mathbf{I}_{2}+\frac{1}{\lambda} \mathbf{M}_{2}-\frac{1}{\lambda} \mathbf{U}_{2}(N-2) \mathbf{M}_{2} \mathbf{Q}_{2} \mathbf{R}_{2}\right]^{-1}$

We can compute the probabilities of system state when there are $n$ customers in the system as follow:

$$
\pi_{2}(n)=\frac{\mathbf{p} \mathbf{U}_{\mathbf{1}}(N) \mathbf{R}_{\mathbf{2}} \mathbf{U}_{2}(N-2) \mathbf{U}_{2}(N-3) \cdots \mathbf{U}_{2}(1) \mathbf{U}_{2}(0)}{\left[\begin{array}{l}
\mathbf{1}+\mathbf{p} \mathbf{U}_{\mathbf{1}}(N) \mathbf{R}_{\mathbf{2}} \mathbf{K}_{2}(N) \boldsymbol{\varepsilon}_{\mathbf{2}}^{\prime}
\end{array}\right]}
$$

The cummulative matrix, denoted as $\mathbf{K}_{\mathbf{2}}(N)$, could be define recursively using following formula:

$$
\begin{aligned}
\mathbf{K}_{\mathbf{2}}(N):= & \mathbf{I}_{\mathbf{2}}+\mathbf{U}_{\mathbf{2}}(N-\mathbf{2})+\mathbf{U}_{\mathbf{2}}(N-\mathbf{2}) \mathbf{U}_{\mathbf{2}}(N-\mathbf{3})+\cdots+\mathbf{U}_{\mathbf{2}}(N-\mathbf{2}) \cdots \mathbf{U}_{\mathbf{2}}(N-n) \\
& +\cdots+\mathbf{U}_{\mathbf{2}}(N-\mathbf{2}) \mathbf{U}_{\mathbf{2}}(N-\mathbf{3}) \cdots \mathbf{U}_{\mathbf{2}}(1) \mathbf{U}_{\mathbf{2}}(0)
\end{aligned}
$$

where $N$ represent an estimated maximum number of customers in the system, whereas $n:=0,1,2, \ldots N$.

The scalar probabilities to represent system state when there are $n \geq 2$ customers and $n \leq 1$ customers, associated for vectors $\pi_{2}(n)$ and $\pi_{1}(n)$ respectively, are defined as follow:

$$
\begin{aligned}
& r(n):=\boldsymbol{\pi}_{2}(n) \boldsymbol{\varepsilon}_{2}^{\prime} \\
& r(n):=\boldsymbol{\pi}_{1}(n) \boldsymbol{\varepsilon}_{1}^{\prime}
\end{aligned}
$$

Finally, the steady state average number of customers in the system can be calculated using the following formula:

$$
\bar{q}_{s}:=\sum_{n=1}^{\infty} n r(n)
$$

Using all above formulas, we can compute queue length of the system and counting average waiting cost for all customers. In this paper, we use the model to analyze service operation for liquid terminal handling CPO cargo in Port of Dumai, Indonesia. Table 1 show observed data during period of August 2010- July 2011.

Table 1. Customer Characteristics of Liquid Terminal

\begin{tabular}{|c|c|c|c|c|c|}
\hline $\begin{array}{c}\text { Ship } \\
\text { Class }\end{array}$ & $\begin{array}{c}\text { Ship Length } \\
\text { (M) }\end{array}$ & $\begin{array}{c}\text { Deadweight Tonnage } \\
\text { (Tons) }\end{array}$ & $\begin{array}{c}\text { Average } \\
\text { Dwt }\end{array}$ & $\begin{array}{c}\text { Mean Service } \\
\text { Time (Hour) }\end{array}$ & $\begin{array}{c}\text { No. Of } \\
\text { Ships }\end{array}$ \\
\hline I & $200-250$ & $58.000-124.500$ & 81.820 & 118,67 & 10 \\
\hline Ii & $150-200$ & $21.900-58.000$ & 39.859 & 68,91 & 186 \\
\hline Iii & $100-150$ & $5.500-21.900$ & 12.358 & 57,43 & 215 \\
\hline Iv & $50-100$ & $500-5.500$ & 3.326 & 47,75 & 122 \\
\hline
\end{tabular}

Using data available on Table 1, as an example, we can define the entrance vector as follows:

$\mathbf{p}=\left[\begin{array}{llll}0,0068 & 0,2237 & 0,4909 & 0,2785\end{array}\right]$.

Using (1), we can compute the following state demotion matrix:

$$
\mathrm{Q}_{2}=\left[\begin{array}{llll}
0.0068 & 0.0000 & 0.0000 & 0.0000 \\
0.1415 & 0.0025 & 0.0000 & 0.0000 \\
0.3308 & 0.0000 & 0.0022 & 0.0000 \\
0.1986 & 0.0000 & 0.0000 & 0.0020 \\
0.0000 & 0.2237 & 0.0000 & 0.0000 \\
0.0000 & 0.2677 & 0.1017 & 0.0000 \\
0.0000 & 0.1645 & 0.0000 & 0.0916 \\
0.0000 & 0.0000 & 0.4909 & 0.0000 \\
0.0000 & 0.0000 & 0.1521 & 0.2228 \\
0.0000 & 0.0000 & 0.0000 & 0.2785
\end{array}\right] .
$$

Whereas the state promotion matrix can be calculate using (2): 


$$
\mathrm{R}_{2}=\left[\begin{array}{llllllllll}
0.0068 & 0.2237 & 0.4909 & 0.2785 & 0.0000 & 0.0000 & 0.0000 & 0.0000 & 0.0000 & 0.0000 \\
0.0000 & 0.0068 & 0.0000 & 0.0000 & 0.2237 & 0.4909 & 0.2785 & 0.0000 & 0.0000 & 0.0000 \\
0.0000 & 0.0000 & 0.0068 & 0.0000 & 0.0000 & 0.2237 & 0.0000 & 0.4909 & 0.2785 & 0.0000 \\
0.0000 & 0.0000 & 0.0000 & 0.0068 & 0.0000 & 0.0000 & 0.2237 & 0.0000 & 0.4909 & 0.2785
\end{array}\right]
$$

Other matrices can be calculated using (3) until (9). Finally, the average number of the customers in the system will be computed using (10) and the result can be summarized as follow:

Table 2. Computational Result of Average Steady State Number of Customers

\begin{tabular}{|c|c|c|c|c|}
\hline $\mathrm{n}$ & $\mathrm{r}(\mathrm{n})$ & $\mathrm{n} \cdot \mathrm{r}(\mathrm{n})$ & $\mathrm{q}_{\mathrm{s}}$ & $\sum \mathrm{r}(\mathrm{n})$ \\
\hline 0 & 0.3174 & 0.0000 & 0.0000 & 0.3174 \\
\hline 1 & 0.2363 & 0.2363 & 0.2363 & 0.5537 \\
\hline 2 & 0.1541 & 0.3082 & 0.5445 & 0.7078 \\
\hline 3 & 0.1006 & 0.3019 & 0.8464 & 0.8085 \\
\hline 4 & 0.0658 & 0.2633 & 1,1097 & 0.8743 \\
\hline 5 & 0.0431 & 0.2156 & 1,3252 & 0.9174 \\
\hline 6 & 0.0283 & 0.1696 & 1,4949 & 0.9457 \\
\hline 7 & 0.0186 & 0.1299 & 1,6248 & 0.9642 \\
\hline 8 & 0.0122 & 0.0976 & 1,7224 & 0.9764 \\
\hline 9 & 0.0080 & 0.0722 & 1,7946 & 0.9844 \\
\hline 10 & 0.0053 & 0.0529 & 1,8474 & 0.9897 \\
\hline 11 & 0.0035 & 0.0383 & 1,8858 & 0.9932 \\
\hline 12 & 0.0023 & 0.0276 & 1,9133 & 0.9955 \\
\hline 13 & 0.0015 & 0.0197 & 1,9331 & 0.9970 \\
\hline 14 & 0.0010 & 0.0140 & 1,9471 & 0.9980 \\
\hline 15 & 0.0007 & 0.0099 & 1,9571 & 0.9987 \\
\hline 16 & 0.0004 & 0.0070 & 1,9641 & 0.9991 \\
\hline 17 & 0.0003 & 0.0049 & 1,9690 & 0.9994 \\
\hline 18 & 0.0002 & 0.0035 & 1,9725 & 0.9996 \\
\hline 19 & 0.0001 & 0.0024 & 1,9749 & 0.9997 \\
\hline 20 & 0.0001 & 0.0017 & 1,9766 & 0.9998 \\
\hline 21 & 0.0001 & 0.0012 & 1,9778 & 0.9999 \\
\hline 22 & 0.0000 & 0.0008 & 1,9786 & 0.9999 \\
\hline 23 & 0.0000 & 0.0006 & 1,9792 & 1,0000 \\
\hline 24 & 0.0000 & 0.0004 & 1,9796 & 1,0000 \\
\hline
\end{tabular}

We can see that, using existing data, the expected average number of customers in the system will reach 1,9796 . If the demand of the terminal increased $100 \%$, while we keep the speed of service for each class of customers, the expected average number of customers will increased to 4,1487. This mean that waiting time of all customers will increased significantly.

In case that the port operator improved his service using better equipments to anticipate the increasing demand, for example increased 2 times of its service speed for each customers, then we can get that the expected number of customer will decrease again to 1.9845 .

\section{CONCLUSION}

If customers in a service system can be categorized into several group based on their service time characteristics, it will be easier to analyze it using linier algebraic queueing theory. In this paper, we used the linier algebraic matrix approach to build a model for liquid terminal operation in the port. The model can be used to analyze the effect of improvement of the service speed and increasing customer arrival in a service 
facility. Using the model we have been succeed to analyze service operation for liquid terminal handling CPO cargo in the port.

In this paper, we defined a server consist of two service stations. Further extension of the model to represent three or more service stations are widely opened, including a formulation for flexible number of service stations to represent terminal operations that can handle different number of customers in a wide variations of shipsize. Further applications of the model are open also for similar service system which exists in other fields, such as manufacturing process, airport chek-in counter, or other public service system.

\section{REFERENCES}

[1] Naor, Paul, On the Regulation of Queue Size by Levying Tolls, Econometrica, 37, 1969, 15-24.

[2] Stidham, Shaler JR., Analysis, Design, and Control of Queueing Systems, Operation Research, 50(1), $2002,197-216$.

[3] Knudsen, Niels Chr., Individual and Social Optimization in a Multiserver Queue with a General Cost-Benefit Structure, Econometrica, 40(3), 1972, 515-528.

[4] Bradford, R., Pricing, routing, and incentive compatibility in multiserver queues, European Journal of Operational Research, 1996, 226-236.

[5] Brouns, Gido A.J.F., Queueing Models with Admission and Termination Control: Monotonicity and Threshold Result, doctoral diss., Universiteitsdrukkerij Technische Universiteit, Eindhoven, The Netherlands, 2003.

[6] Ahn, Hyun-soo, and Mark E. Lewis, Flexible Server Allocation and Customer Routing Policies for Two Parallel Queues when Service Rates are not Additive, 2011.

[7] Lipsky, L., Queueing Theory - A Linear Algebraic Approach - Second Edition (NY: Springer Science + Business Media, 2009). 